\title{
Bioquímica plasmática de cascavéis (Caudisona durissa LINNAEUS, 1758) em cativeiro
}

\author{
Blood biochemical parameters from captivity rattlesnake (Caudisona durissa LINNAEUS, 1758)
}

\author{
Wellington Bandeira da Silva ${ }^{\mathrm{I}^{*}}$ Rodrigo Machado Soares $^{\mathrm{II}}$ Claudio Machado ${ }^{\mathrm{III}}$ \\ Isabel Maria Alexandre Freire ${ }^{\mathrm{IV}}$ Luiz César Cavalcanti Pereira da Silva ${ }^{\mathrm{IV}}$ \\ Silvia Bahadian MoreiraI ${ }^{I}$ Daphne Wrobel GoldbergI Nádia Regina Pereira Almosny ${ }^{I}$
}

\section{RESUMO}

Acidentes com seres humanos envolvendo a espécie Caudisona durissa possuem a mais alta taxa de letalidade dentre os viperídeos brasileiros. Ressalta-se então a importância para a saúde pública da criação dessa espécie em cativeiro para produção de soro antiofídico. No entanto, essa atividade ainda hoje encontra alguns desafios como a instalação de doenças no plantel, evidenciando a importância de estudos sobre a fisiologia de serpentes. Dessa forma, foram realizadas análises de bioquímica plasmática em 53 serpentes da espécie Caudisona durissa, mantidas em cativeiro. Foram utilizadas amostras de plasma com heparina e as dosagens bioquímicas realizadas em aparelho automatizado (Ciba Corning - Express Plus $\left.{ }^{\circledR}\right)$. Os resultados e seus respectivos desvios-padrões foram: uréia $-1,32 \mathrm{mg} d L^{-1}( \pm 1,1)$; ácido úrico - 2,08mg $d L^{-1}( \pm 1,4)$; creatinina - 0,52mg $d L^{-1}( \pm 0,2)$; proteina total - 3,7g $d L^{-1}( \pm 0,7)$; albumina - 1,62g $d L^{-1}( \pm 0,4)$; globulinas - 2,08g $d L^{-1}( \pm 0,5)$; cálcio - 15,25mg $d L^{-1}( \pm 2,8)$; fósforo $4,61 \mathrm{mg}^{-1} L^{-1}( \pm 1,9)$; colesterol - 171,58mg $d L^{-1}( \pm 52,7)$; triglicerideos - 19,29mg $d L^{-1}( \pm 14,3)$; fosfatase alcalina $31,04 U L^{-1}( \pm 12,4)$; aspartato aminotransferase $(A S T)-22,25 U$ $L^{-1}( \pm 11,4)$; alanina aminotransferase $(A L T)-7,11 U L^{-1}( \pm 5,4)$ e Amilase - 1385,23U $L^{-1}( \pm 568,7)$. Foram calculados os seguintes indices: relação uréia/creatinina - 2,5 e relação cálcio/fósforo - 3,3. O conjunto de resultados das análises bioquímicas do sangue das cascavéis em cativeiro pode servir como referência para apoio diagnóstico na espécie estudada e para outros trabalhos com o mesmo caráter de observação.

Palavras-chave: Caudisona durissa, cascavel, bioquímica, réptil.

\begin{abstract}
Human accidents involving rattlesnake Caudisona durissa have the highest fatality rate among the Brazilian Viperidae family. Breeding this specie in captivity in order to produce antivenoms is very important to public health. Nevertheless, there are some challenges that this activity must face, like the onset of diseases on the breeding stock, justifying the importance of studying these snakes' physiology. Blood biochemical analysis of 53 Caudisona durissa was performed using plasma samples in an automated equipment (Ciba Corning - Express Plus $\left.{ }^{\circledR}\right)$. Mean values and its respective Standard deviations are: blood urea nitrogen (BUN) $-1.32 \mathrm{mg}$ $d L^{-1}( \pm 1.1)$; uric acid $-2.08 \mathrm{mg} d L^{-1}( \pm 1.4)$; creatinine $-0.52 \mathrm{mg}$ $d L^{-1}( \pm 0.2)$; total protein - $3.7 g d L^{-1}( \pm 0.7)$; albumin - $1.62 g$ $d L^{-1}( \pm 0.4) ;$ globulins - $2.08 \mathrm{~g} d L^{-1}( \pm 0.5)$; calcium - $15.25 \mathrm{mg}$ $d L^{-1}( \pm 2.8)$; phosphorus - 4.61mg $d L^{-1}( \pm 1.9)$; cholesterol -

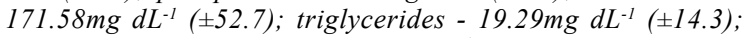
alkaline phosfatase - $31.04 U L^{-1} \quad( \pm 12.4) ;$ aspartate aminotransferase (AST) - 22.25U $L^{-1}( \pm 11.4)$; alanin aminotransferase (ALT) $7.11 \mathrm{UL}^{-1}( \pm 5.4)$; amylases - $1385.23 \mathrm{U}$ $L^{-1}( \pm 568.7)$. The following indexes were calculated: BUN/ creatinine ratio - 2.5 and calcium/phosphorus ratio -3.3. Rattlesnake blood biochemical results above could be used as reference in clinical evaluation for captivity $\boldsymbol{C}$. durissa.
\end{abstract}

Key words: Caudisona durissa, rattlesnake, blood biochemistry.

\section{INTRODUÇÃO}

O gênero Caudisona está representado no Brasil por uma única espécie: Caudisona durissa. É

IPrograma de Pós-graduação em Medicina Veterinária (Clínica e Reprodução Animal), Faculdade de Medicina Veterinária, Universidade Federal Fluminense (UFF), Rua Vital Brazil Filho, 64, 24230-360, Niterói, RJ, Brasil. E-mail: wellban@yahoo.com.br.*Autor para correspondência.

"Faculdade de Ciências Biológicas, Universidade Gama Filho (UGF), Piedade, RJ, Brasil.

IIIInstituto Vital Brazil, Divisão de Herpetologia, Niterói, RJ, Brasil.

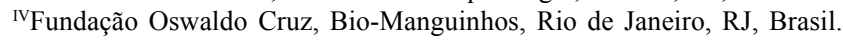


uma espécie predadora que habita os cerrados do Brasil Central, as regiões áridas e semi-áridas do Nordeste e os campos e áreas abertas do Sul, Sudeste e Norte e, apesar de ser responsável apenas por cerca de $8 \%$ dos acidentes ofídicos no país, possui a mais alta taxa de letalidade dentre os viperídeos brasileiros (MELGAREJO, 2003; ARAÚJO et al., 2003). Com o crescimento desordenado das cidades e fragmentação das áreas naturais, ocorre o aumento da densidade populacional desses animais em áreas urbanas, resultando no aumento das notificações de acidentes (ARAÚJO et al., 2003; BASTOS et al., 2005). Nesse sentido, a criação de serpentes peçonhentas em cativeiro com a finalidade de produção de soro antiofídico é de grande importância para a saúde pública.

Além de contribuir para o conhecimento da biologia da espécie, a determinação de parâmetros fisiológicos de uma população saudável mantida em cativeiro é fundamental para o manejo de um serpentário, já que pode servir como indicador de saúde do indivíduo. As análises bioquímicas são úteis no auxílio ao diagnóstico, no acompanhamento clínico e no prognóstico de diversas moléstias que acometem os animais. Utilizado com frequência em outras classes de vertebrados, essas análises ainda carecem de dados disponíveis na literatura para répteis. Assim, este trabalho tem como objetivo testar métodos analíticos, utilizados em exames bioquímicos de animais domésticos em plasma de cascavéis (Caudisona durissa) mantidas em cativeiro e estabelecer um perfil dos valores de bioquímica plasmática para esses animais.

\section{MATERIAL E MÉTODOS}

Foram realizadas análises de bioquímica plasmática em 53 exemplares da espécie Caudisona durissa, mantidas em cativeiro na Divisão de Herpetologia do Instituto Vital Brazil (Niterói, RJ). Os animais foram selecionados após constatação de sanidade por exame físico e observação de agressividade normal para a espécie e seu peso médio foi de 665 gramas. As amostras de sangue foram coletadas nos meses de outubro de 2007 (30 exemplares) e janeiro de 2009 ( 23 exemplares). Os animais utilizados foram retirados da rotina de extração de veneno por um período de quatro meses antes das coletas.

Quarenta dias antes das coletas, preconizou-se o uso de Ivermectina (Ivomec ${ }^{\circledR}$ ), por via subcutânea (SC), em duas doses com intervalo de 15 dias; Levamizole (Ripercol ${ }^{\circledR}$ ), SC, em dose única de $8 \mathrm{mg}$ $\mathrm{kg}^{-1}$; e Fipronil 10\% (Frontline ${ }^{\circledR}$ Spray) para controle de endoparasitas e ectoparasitas, seguindo as instruções de CARPENTER et al. (2004).

Os animais foram acondicionados individualmente em caixas retangulares de polipropileno leitoso $(50 \times 35 \times 16 \mathrm{~cm}-$ comprimento $\mathrm{x}$ largura $x$ altura), devidamente identificadas e mantidas dentro de uma sala a temperatura ambiente $\left(25-30^{\circ} \mathrm{C}\right)$. Eles foram regularmente alimentados uma vez ao mês com dois camundongos originários do biotério da própria instituição e com livre acesso a água.

Após a contenção física, foi realizada a coleta de $0,5 \mathrm{~mL}$ de sangue de cada serpente através de punção da veia coccígea ventral, utilizando-se agulha $20 \times 0,55 \mathrm{~mm}$ e seringa de $1 \mathrm{~mL}$. As amostras foram armazenadas em frasco plástico de fundo cônico $\left(\right.$ Eppendorf $\left.^{\mathbb{E}}\right)$ previamente rinçado com heparina sódica 5.000UI mL ${ }^{-1}$ (Liquemine $^{\circledR}$ ) e centrifugados a 5.000rpm durante 5 minutos, segundo ALMOSNY \& MONTEIRO (2007), para a obtenção do plasma (aproximadamente $0,3 \mathrm{~mL}$ por amostra).

As dosagens bioquímicas foram realizadas em equipamento automatizado (Ciba Corning - Express Plus $^{\circledR}$ ) e o controle de precisão de análise foi realizado previamente com soros controles normal e patológico (Quantnorm ${ }^{\circledR}$ e Quantalt ${ }^{\circledR}$ Biotecnica). Utilizaram-se reativos comerciais $\left(\right.$ Biotecnica $^{\circledR}$ ) e as amostras foram processadas segundo as recomendações do fabricante. As dosagens de proteína total, albumina e cálcio foram determinadas por métodos colorimétricos. As dosagens de ácido úrico, colesterol e triglicerídeos foram realizadas utilizando-se métodos enzimáticos colorimétricos. O método cinético colorimétrico foi utilizado para medir a atividade de fosfatase alcalina, amilase e a concentração de creatinina. A concentração de uréia foi mensurada por método cinético enzimático ultravioleta. As atividades de alanina aminotransferase (ALT) e de aspartato aminotransferase (AST) foram determinadas por método cinético ultravioleta e a concentração de fósforo pelo método do molibdato ultravioleta. A concentração das globulinas foi obtida subtraindo-se o valor da albumina do valor das proteínas totais. Em função do reduzido volume de sangue obtido, da variação do requerimento de amostra necessária para cada análise e da necessidade de eventuais repetições para confirmação de resultados discrepantes, o número de amostras para cada analito foi diferente devido ao esgotamento do plasma a ser analisado.

Foram calculadas a relação uréia/creatinina e cálcio/fósforo para os valores observados neste trabalho e os mesmos índices para os valores apresentados em TROIANO et al. (2001) e em KOLESNIKOVAS et al. (2001), a título de comparação. 
A conversão para o sistema internacional de unidades foi realizada segundo CUBAS (2007), visando a uma comparação com a literatura existente.

Foram calculadas médias e desvio-padrão de cada parâmetro bioquímico analisado.

\section{RESULTADOS E DISCUSSÃO}

Os resultados das concentrações dos analitos estão dispostos na tabela 1 .

Os valores médios de uréia no presente trabalho $\left(1,32 \mathrm{mg} \mathrm{dL}^{-1}\right)$ estão de acordo com CAMPBELL (2006), que relata o fato de que répteis terrestres possuem a concentração normal de uréia abaixo de $15 \mathrm{mg} \mathrm{dL}^{-1}$, por serem uricotélicos. Esse seria um mecanismo para elevar a osmolaridade plasmática e assim reduzir a perda de água corporal. Os valores plasmáticos de uréia geralmente são pobres indicadores de doença renal em répteis, com exceção dos répteis aquáticos, que excretam primariamente a uréia como catabólito protéico. Já a média dos valores de ácido úrico encontrada $\left(2,08 \mathrm{mg} \mathrm{dL}^{-1}\right)$ foi pouco maior que a média encontrada para uréia, o que também foi observado por KOLESNIKOVAS et al. (2001), comprovando a natureza uricotélica do gênero Caudisona, sendo este um réptil terrestre. KOLESNIKOVAS et al. (2001) demonstraram valores médios de uréia $\left(3,19 \mathrm{mg} \mathrm{dL}^{-1}\right)$ e ácido úrico $\left(5,8 \mathrm{mg} \mathrm{dL}^{-1}\right)$ maiores do que os apresentados no presente trabalho, com a ressalva de que utilizaram a média dos valores observados para dois gêneros diferentes, Caudisona e Bothrops. De forma geral, a excreção de compostos nitrogenados está relacionada à disposição de água no meio (SCHIMIDT-NIELSEN, 1999). Por serem incapazes de concentrar urina, devido à ausência da alça de Henle em seus rins, os répteis terrestres não conseguem eliminar resíduos protéicos como amônia e uréia sem a perda concomitante de água. Dessa forma, 50 a $85 \%$ dos produtos nitrogenados são excretados em formas pouco solúveis e semi-sólidas, tais como o ácido úrico (ALMOSNY \& MONTEIRO, 2007). Vale realçar que o aumento dos níveis plasmáticos de uréia e ácido úrico devem ser avaliados com cautela, pois a elevação de um desses parâmetros pode ser decorrente de dietas ricas em proteínas. Em répteis carnívoros, tal como a Caudisona, a concentração de ácido úrico pode ser elevar em até duas vezes no período pós-prandial (ALMOSNY \& MONTEIRO, 2007).

Tabela 1 - Média e desvio-padrão dos valores de bioquímica plasmática para serpentes da espécie Caudisona durissa, expressos em unidades convencionais e no sistema internacional de unidades.

\begin{tabular}{llll}
\hline Analito & Unidade Convencional & Sistema Internacional de Unidades & $\mathrm{n}$ \\
\hline Uréia & $1,32 \mathrm{~g} \mathrm{dL}^{-1}( \pm 1,1)$ & $0,47 \mathrm{mmol} \mathrm{L}^{-1}( \pm 0,40)$ & 47 \\
Ácido Úrico & $2,08 \mathrm{mg} \mathrm{dL}^{-1}( \pm 1,4)$ & $0,12 \mathrm{mmol} \mathrm{L}^{-1}( \pm 0,08)$ & 27 \\
Creatinina & $0,52 \mathrm{mg} \mathrm{dL}^{-1}( \pm 0,2)$ & $0,05 \mu \mathrm{mol} \mathrm{L}^{-1}( \pm 0,02)$ & 50 \\
Proteína Total & $3,7 \mathrm{~g} \mathrm{dL}^{-1}( \pm 0,7)$ & $37,0 \mathrm{~g} \mathrm{~L}^{-1}( \pm 7,0)$ & 51 \\
Albumina & $1,62 \mathrm{~g} \mathrm{dL}^{-1}( \pm 0,4)$ & $16,2 \mathrm{~g} \mathrm{~L}^{-1}( \pm 4,0)$ & 51 \\
Globulinas & $2,08 \mathrm{~g} \mathrm{dL}^{-1}( \pm 0,5)$ & $20,8 \mathrm{~g} \mathrm{~L}^{-1}( \pm 3,0)$ & 51 \\
Cálcio & $15,25 \mathrm{mg} \mathrm{dL}^{-1}( \pm 2,8)$ & $3,81 \mathrm{mmol} \mathrm{L}^{-1}( \pm 0,70)$ & 33 \\
Fósforo & $4,61 \mathrm{mg} \mathrm{dL}^{-1}( \pm 1,9)$ & $1,49 \mathrm{mmol}^{-1} \mathrm{~L}( \pm 0,61)$ & 34 \\
Colesterol & $171,58 \mathrm{mg} \mathrm{dL}^{-1}( \pm 52,7)$ & $4,44 \mathrm{mmol} \mathrm{L}^{-1}( \pm 1,36)$ & 33 \\
Triglicerídeos & $19,29 \mathrm{mg} \mathrm{dL}^{-1}( \pm 14,3)$ & $0,22 \mathrm{mmol} \mathrm{L}^{-1}( \pm 0,16)$ & 34 \\
Fosfatase Alcalina & $31,04 \mathrm{U} \mathrm{L}^{-1}( \pm 12,4)$ & $31,04 \mathrm{U} \mathrm{L}^{-1}( \pm 12,4)$ & 51 \\
AST/TGO & $22,25 \mathrm{U} \mathrm{L}^{-1}( \pm 11,4)$ & $22,25 \mathrm{U} \mathrm{L}^{-1}( \pm 11,4)$ & 52 \\
ALT/TGP & $7,11 \mathrm{U} \mathrm{L}^{-1}( \pm 5,4)$ & $7,11 \mathrm{U} \mathrm{L}^{-1}( \pm 5,4)$ & $1385,23 \mathrm{U} \mathrm{L}^{-1}( \pm 568,7)$ \\
Amilase & $1385,23 \mathrm{U} \mathrm{L}^{-1}( \pm 568,7)$ & & 26 \\
\hline
\end{tabular}

$\mathrm{n}$ - número de amostras em que foram realizadas dosagens dos analitos.

AST - Aspartato aminotransferase; ALT - Alanino aminotransferase.

Ciência Rural, v.40, n.12, dez, 2010. 
A determinação dos valores séricos de creatinina é a forma mais comum de se avaliar a função renal em mamíferos (ALMOSNY \& MONTEIRO, 2007). No entanto, em répteis, o nível encontrado geralmente é baixo e não parece ser um bom indicador de doença renal, (CAMPBELL, 2006). Os valores de creatinina encontrados neste trabalho $\left(0,52 \mathrm{mg} \mathrm{dL}^{-1}\right)$ foram menores que os encontrados por KOLESNIKOVAS et al. (2001) (1,04mg dL $\left.\mathrm{m}^{-1}\right)$. Segundo ALMOSNY \& MONTEIRO (2007), a relação uréia/creatinina pode ser usada como indicador de catabolismo proteico e na diferenciação entre insuficiência renal e pré-renal. Apesar de essa abordagem ainda carecer de padronização para a espécie, observou-se uma proximidade da relação uréia/creatinina observados neste trabalho $(2,5)$, com o valor encontrado por KOLESNIKOVAS et al. (2001) $(3,1)$ e mesmo por TROIANO et al. (2001) (4,0), que descrevem valores de creatinina e uréia maiores.

Os valores de cálcio observados neste trabalho $\left(15,25 \mathrm{mg} \mathrm{L}^{-1}\right.$ ou $\left.3,81 \mathrm{mmol} \mathrm{L}^{-1}\right)$ foram semelhantes aos observados por KOLESNIKOVAS et al. (2001) $\left(15,13 \mathrm{mg} \mathrm{dL}^{-1}\right)$ e maiores do que os encontrados por TROIANO et al. (2001) $\left(2,03 \mathrm{mmol} \mathrm{L}^{-1}\right)$. Para fósforo, a média observada $\left(4,61 \mathrm{mg} \mathrm{dL}^{-1}\right.$ ou $1,49 \mathrm{mmol} \mathrm{L}^{-1}$ ) foi maior do que o descrito por KOLESNIKOVAS et al. (2001) $\left(3,29 \mathrm{mg} \mathrm{dL}^{-1}\right)$ e pouco maior do que o observado por TROIANO et al. (2001) $\left(1,25 \mathrm{mmol} \mathrm{L}^{-1}\right)$. Apesar de os três trabalhos apresentarem relação cálcio/fósforo acima de 1 , os valores encontrados neste trabalho $(3,3)$ se diferenciaram do observado por TROIANO et al. (2001) $(1,6)$ e KOLESNIKOVAS et al. $(2001)(4,6)$. Segundo DIVERS (2000a), o cálcio e o fósforo são minerais que atuam em diversas funções fisiológicas e a determinação de sua concentração plasmática é importante no diagnóstico de várias doenças. A relação cálcio/fósforo é provavelmente um bom indicador de doença renal em répteis. Em animais sadios, essa relação está geralmente acima de 1, sendo a doença renal caracterizada por valores menores.

Embora este trabalho apresente valores de proteína plasmática total próximos ao limite inferior descrito por CAMPBELL (2006) para répteis em geral ( 3 a $\left.8 \mathrm{mg} \mathrm{dL}^{-1}\right)$, a média observada $\left(3,70 \mathrm{~g} \mathrm{dL}^{-1}\right)$ corroborou com o descrito por KOLESNIKOVAS et al. (2001) $\left(3,71 \mathrm{mg} \mathrm{dL}^{-1}\right)$ e TROIANO et al. (2001) $\left(3,79 \mathrm{mg} \mathrm{dL}^{-1}\right)$. Comportamento semelhante se observou para os valores de albumina e globulinas. Hipoproteínemia em répteis está relacionada à má nutrição, má absorção, às enteropatias e às doenças renais crônicas e hepáticas (ALMOSNY \& MONTEIRO, 2007). No entanto, a ausência de sinais nos animais estudados sugerem a normalidade dos valores observados.
Os valores médios de ALT observados neste trabalho $\left(7,11 \mathrm{U} \mathrm{L}^{-1}\right)$ foram menores que os relatados por TROIANO et al. (2001) $\left(13,00 \mathrm{U} \mathrm{L}^{-1}\right)$ e por KOLESNIKOVAS et al. (2001) (11,32U L $\left.\mathrm{U}^{-1}\right)$. No entanto, os valores limítrofes de ALT, descritos neste trabalho, cruzaram-se com os valores limítrofes observados na literatura. Em relação à enzima $\mathrm{AST}$, o presente estudo observou valor médio $\left(22,25 \mathrm{U} \mathrm{L}^{-1}\right)$, próximo aos achados de TROIANO et al. $\left(20,00 \mathrm{U} \mathrm{L}^{-1}\right)$ e superior ao que foi encontrado por KOLESNIKOVAS et al. (2001) (9,73U $\left.\mathrm{L}^{-1}\right)$. As atividades séricas de ALT e AST são elevadas no tecido hepático de répteis e o seu aumento pode sugerir doença hepatocelular. Todavia, a AST não é considerada um sensível indicador hepático por estar presente também em outros tecidos nos répteis (ALMOSNY \& MONTEIRO, 2007; DIVERS, 2000b).

A fosfatase alcalina pode indicar atividade osteoblástica, no entanto não se mostra uma enzima órgão específica, já que está presente em diversos tecidos de répteis (ALMOSNY \& MONTEIRO, 2007). $\mathrm{O}$ valor médio observado neste trabalho $\left(31,04 \mathrm{U} \mathrm{L}^{-1}\right)$ foi superior ao observado por TROIANO et al. (2001) $\left(17,1 \mathrm{U} \mathrm{L}^{-1}\right)$ e está dentro do largo intervalo descrito por KOLESNIKOVAS et al. (2001) (11,34 a 195,42U L-1).

Os valores séricos de colesterol e triglicerídeos podem estar fisiologicamente aumentados, devido à vitelogênese em fêmeas ou patologicamente em decorrência de uma lipidose hepática (DIVERS, 2000c). A média dos valores de colesterol encontrados neste trabalho $\left(171,58 \mathrm{mg} \mathrm{dL}^{-1}\right.$ ou 4,44mmol L-1) está dentro da variação observada por KOLESNIKOVAS et al. (2001) (239,8 $\left.\mathrm{mg} \mathrm{dl}^{-1}\right)$ e foram superiores aos valores observados por TROIANO et al. (2001) $\left(2,07 \mathrm{mmol} \mathrm{L}^{-1}\right)$. Entretanto, os valores de triglicerídeos $\left(19,29 \mathrm{mg} \mathrm{dL}^{-1}\right.$ ou $\left.0,22 \mathrm{mmol} \mathrm{L}^{-1}\right)$ se mostraram menores do que o observado por TROIANO et al.(2001) $\left(2,80 \mathrm{mmol} \mathrm{L}^{-1}\right)$ e não foi estudado no trabalho de KOLESNIKOVAS et al. (2001).

Segundo DIVERS (2000c), a amilase é uma enzima pancreática comumente usada no diagnóstico de lesão no pâncreas em répteis, no entanto, neste trabalho, observou-se grande variação de desvio padrão, o que não permitiu correlação com o estado de saúde dos animais. Além disso, esta enzima parece ser ainda pouco estudada para a espécie.

\section{CONCLUSÃO}

De forma geral, a utilização de metodologia analítica e reagentes consagrados para exames bioquímicos em animais domésticos se mostrou adequada para determinação dos parâmetros analisados em cascavéis, com exceção da dosagem de amilase. 
As relações uréia/creatinina e cálcio/fósforo apresentaram valores próximos ao descrito pela literatura, sugerindo a validade desses índices no diagnóstico diferencial de patologias renais em cascavéis.

Estudos posteriores serão necessários para que haja um melhor entendimento dos parâmetros bioquímicos e sua utilização com maior precisão na clínica da espécie. No entanto, espera-se que o conjunto de resultados observados neste trabalho possa servir como referência para outros trabalhos com o mesmo caráter de observação.

\section{AGRADECIMENTOS}

Ao Centro de Apoio ao Diagnóstico Veterinário (CAD - Tijuca/Rio de Janeiro), por disponibilizar os equipamentos e os reagentes para realização deste trabalho.

Gostaríamos de agradecer à Coordenação de Aperfeiçoamento de Pessoal de Nível Superior (CAPES), pelo suporte financeiro cedido a este projeto.

Aos profissionais do Instituto Vital Brazil, por permitir a execução deste trabalho e pela ajuda na contenção dos animais.

\section{REFERÊNCIAS}

ALMOSNY, N.R.P.; MONTEIRO, A.M. Patologia clínica. In: CUBAS, Z.S. et al. Tratado de animais selvagens: medicina veterinária. São Paulo: Roca, 2007. Cap59, p.939-367.

ARAÚJO, F.A.A. et al. Epidemiologia dos acidentes por animais peçonhentos. In: CARDOSO, J.L.C. et al. Animais peçonhentos no Brasil - Biologia, clínica e terapêutica dos acidentes. São Paulo: SARVIER, 2003. Cap.2, p.6-12.

BASTOS, E.G.M. et al. Records of the rattlesnakes Crotalus durissus terrificus (Laurenti) (Serpentes, Viperidae) in the State of Rio de Janeiro, Brazil: a possible case of invasion facilitated by deforestation. Revista Brasileira de Zoologia, v.22, n.3, p.812-815, 2005. Disponível em: <http://www.scielo.br/pdf/ rbzool/v22n3/26210.pdf>. Acesso em: 2 nov. 2010. doi: 10.1590/S0101-81752005000300047.
CAMPBELL, T.W. Clinical pathology of reptiles. In: MADER, D.R. (Ed.). Reptile medicine and surgery. Missouri: Saunders Elsevier, 2006. Cap.22, p.490-532.

CARPENTER J.W. et al. Antiparasitic agents used in reptiles. In:_ Exotic animal formulary. 3.ed. Philadelphia: Saunders, 2004. p.51-58.

CUBAS, Z.S. Fatores de conversão entre unidades convencionais e internacionais. In: CUBAS, Z.S. et al. Tratado de animais selvagens: medicina veterinária. São Paulo: Roca, 2007. p.1308-1310.

DIVERS, S.J. Reptilian metabolic disorders. In: FUDGE, A.M. Laboratory medicine - avian and exotic pets. Philadelphia: Saunders, 2000a. Cap.24, p.210-216.

DIVERS, S.J. Liver and gastrointestinal testing. In: FUDGE, A.M. Laboratory medicine - avian and exotic pets. Philadelphia: Saunders, 2000b. Cap.23, p.205-210.

DIVERS, S.J. Reptilian renal and reproductive disease diagnosis. In: FUDGE, A.M. Laboratory medicine - avian and exotic pets. Philadelphia: Saunders, 2000c. Cap.25, p.217-222.

KOLESNIKOVAS, C.K.M. et al. Biochemical blood profile of Brazilian viperidae snakes kept in captivity. In: REUNIÃO CIENTÍFICA ANUAL DO INSTITUTO BUTANTAN, 2001, São Paulo- SP. Memórias do Instituto Butantan, v.59, p.141-142, 2001.

MELGAREJO, A.R. Serpentes peçonhentas do Brasil. In: CARDOSO, J.L.C. et al. Animais peçonhentos no Brasil Biologia, clínica e terapêutica dos acidentes. São Paulo: Sarvier, 2003. Cap.4, p.36-61.

SCHIMIDT-NIELSEN, K. Excreção. In: Fisiologia animal - adaptação e meio ambiente. São Paulo: Santos, 1999. Cap.9, p.353-392.

TROIANO, J.C. et al. Blood biochemical profile of the south american rattlesnakes (Crotalus durissus terrificus) in Captivity. Journal of Venomous Animals and Toxins, v.7, n.2, p.183-189, 2001. Disponível em:<http:// www.scielo.br/scielo.php? script $=$ sci arttext\&pid $=$ S0104$79302001000200004 \& \operatorname{lng}=$ en $\& n r m=i$ iso $\& \operatorname{tn} g=e n>$. Acesso em: 2 nov. 2010. doi: 10.1590/S0104-79302001000200004. 